\title{
Effects of Repeated Extracorporeal Shock Wave on Kidney Apoptosis of Normal and Diabetic Rat
}

\author{
Vicente M. Kira, Djalma J. Fagundes, Cesar O. P. Bandeira, Oskar Kaufman, Anna T. N. \\ Fagundes, Valdemar Ortiz
}

\author{
Division of Urology and Division of Surgical Technique and Experimental Surgery, Department of \\ Surgery, Paulista School of Medicine, Federal University of Sao Paulo, UNIFESP, Sao Paulo, SP, \\ Brazil
}

\begin{abstract}
Objective: To assess the effect of repeated extracorporeal shock waves (ESW) on the apoptosis of renal parenchyma in normal and diabetic rats by the caspase-3 expression.

Materials and methods: 80 rats were assigned for ESW (Direx Tripter X1 ${ }^{\circledR}$ - $14 \mathrm{KVA}$ ) to one of three groups with $50 \%$ rats with diabetes (A) and 50\% normal (B) in each: G1 $(n=40)$ : no ESW; G2 $(n=20)$ : one ESW; G3 $(n=20)$ : two ESWin an interval of 14 days. The animals were sacrificed 3 days after the ESW and samples of the renal parenchyma were histologically prepared, stained by the caspase-3 cleavage immunohistochemical method, and the apoptotic index (ApIn) was calculated by computer program Image Pro-Plus V 4,5,1 Media Cybernetics ${ }^{\circledR}($ ApIn = number of apoptotic cells in the microscopic section / total cells in the microscopic section).

Results: There was no statistical difference in ApIn of normal rats with one ESW $(8.4 \pm 0.2)$ or two ESW $(9.1 \pm 0.3)$ and both were similar to the control group with no ESW (7.9 \pm 0.2$)$. The ApIn of diabetic rats with one ESW $(7.4 \pm 0.3)$ was similar to the normal rats with one or two ESW. On the other hand, the ApIn was statistically higher in diabetic rats with two ESW (11.9 $\pm 0.4)$ than in diabetic rats with one ESW (7.4 \pm 0.3$)$.

Conclusions: The ApIn of normal rats with a single (2,000 SW) or repeated ESW (4,000 SW) was similar to the control animals without any ESW. In diabetic rats, the repeated ESW (4,000 SW) may result in an additional risk of tissue damage expressed by the higher ApIn.
\end{abstract}

Key words: kidney; lithotripsy; high-energy shock waves; diabetes; rats; apoptosis

Int Braz.J Urol. 2008; 34: $91-6$

\section{INTRODUCTION}

Extracorporeal shock wave lithotripsy (ESWL) has become a routine method for the treatment of renal and ureteral calculi (1). Recovery of renal tissue damage and reduced functioning in both kidneys occurred in a few days (2). However, occa- sionally multisequential ESWL treatments and highenergy shock waves are required to treat large calculi $(2,3)$. There is no consensus about the interval times between treatments or the predictive grade of renal parenchyma injury (3-6). Despite the current use of ESWL, there is a lack of definitive knowledge about injury caused by re-treatment within a short time 
period, and so the treatment was performed in an empirical way (3-6).

On the other hand, the prevalence of renal stones in the diabetic population has recently been estimated at $21 \%$, i.e. more than twice the prevalence of stones in the general population (7). The association of diabetic nephropathy and urolithiasis has a potential risk of impairment of the renal structure or function, and how much that risk may be increased by ESW is unknown (8).

The renal functional response to lithotripsy has shown that ESWL triggers vasoconstriction in the kidney, and the cavitation that occurs in kidney tissue is a critical issue in determining the mechanisms of tissue injury (9). ESWL damages the tissue mainly through vascular injury and subsequent hemorrhage focus in the capsular and glomerular areas (8). Both vasoconstriction and hemorrhage lead to tissue hypoxia and these promote necrosis. Tissue hypoxia that occurs under this pathological condition is also a critical factor in the development of apoptosis (10). The primary executioners of apoptosis are the caspase family, which are activated after acute kidney injuries (11). Caspase-3 plays a key role in apoptosis, but the involvement of apoptosis and caspase- 3 in mediating ESWL injury are not fully defined (11).

The objective of this study was to assess the effects of repeated extracorporeal shock waves (ESW) on the apoptosis of renal parenchyma in normal and diabetic rats using the caspase- 3 expression.

\section{MATERIALS AND METHODS}

The experimental protocol was approved by the Ethics Committee of the Federal University of São Paulo. All the procedures strictly followed existing regulations about animal experimentation. All surgical procedures and ESW were done under general anesthesia (intramuscular via) with ketamine $\left(60 \mathrm{mg} \cdot \mathrm{Kg}^{-1}\right)$ and xylazine $\left(5 \mathrm{mg} \cdot \mathrm{Kg}^{-1}\right)$.

Initially, a hundred male Wistar rats weighing between 250-300g were subjected to laparotomy and a radiopaque device (a piece of radiopaque surgical gauze, $0.2 \mathrm{~cm}$ in diameter) was sutured at the adipose tissue situated in the anterior face of the right renal pelvis near the renal parenchyma. The standard position of the X-ray marker was confirmed through the simple postero-anterior radiographic method.

On the 14th postoperative day, fifty animals were induced to diabetes (12) with an intravenous dose of alloxan (45 mg. $\left.\mathrm{Kg}^{-1}\right)$, while the other fifty animals received an intravenous dose of saline solution. The glycemia values were evaluated in all animals from both groups on the 6th and 30th days after the intravenous alloxan or saline solution, always at $4 \mathrm{pm}$. One day before the first ESW, the 24 hours urine was collected for the albumin values $(\mu \mathrm{g} / \mathrm{L})$ dosage. Forty animals with persistent glycemia values over $200 \mathrm{mg} / \mathrm{dL}$ (alloxan) were randomly allocated to group A while another forty bearing normal glycemia values (saline) were randomly allocated to group B.

Using a Direx Tripter X1® litotriptor - 14 KVA (13) - the focus system was obtained by means of acoustic lens and contact with the animal skin through a bag containing water and gel. Previously to the adjustment of the focus system, the radiopaque device was located by fluoroscope monitor. The ESW was focused about $0.5 \mathrm{~cm}$ laterally to the radiopaque device and 2,000 shock waves were applied during each session.

For ESW treatment the animals were assigned to one of three groups, 30 days after the diabetes induction. Groups A1 $(n=20)$ and B1 $(n=20)$ did not receive ESW and were sacrificed on the $3^{\text {rd }}(\mathrm{n}=$ $10)$ or the $17^{\text {th }}$ day $(n=10)$. They served as references for absence of ESW injury. The animals from A2 $(n=10)$ and B2 $(n=10)$ received 2,000 ESW on the $1^{\text {st }}$ day and were sacrificed on the $3^{\text {rd }}$; the animals from $A 3(n=10)$ and $B 3(n=10)$ received 2,000 ESW on the $1^{\text {st }}$ day and another 2,000 ESW on the $14^{\text {th }}$ day and were sacrificed on the $17^{\text {th }}$ day.

Seventy-two hours after the last ESW, the animals were submitted to a right nephrectomy and 3 samples $(0.5 \times 0.5 \mathrm{~cm})$ of renal parenchyma (cortex and medullar) were collected from the area close to the radiopaque device $(0.5 \mathrm{~cm})$ where the ESW was focused. These kidney specimens were fixed in $10 \%$ formalin solution, embedded in paraffin wax and the histological sections $(5 \mu \mathrm{m})$ were stained by the imunohistochemical method of activated caspase- 3 (Cell Signaling Technology, code 9661, USA). A his- 
tological evaluation was undertaken by an independent pathologist who had no prior knowledge of the experimental groups from which the specimens were derived. From the three samples of each animal three histological sections were randomly chosen and microscopically examined. The twenty best areas were selected for morphometric study. The images were captured with a high-resolution AxioCam ${ }^{\circledR}$ camera coupled with a Carl Zeiss Axilab ${ }^{\circledR}$ light microscope. A negative mask of the immunohistochemical stained cells by caspase-3 was made from each microscopic section. The apoptotic index (ApIn) was calculated by the Image Pro-Plus V computer program. 4, 5, 1 Media Cybernetics ${ }^{\circledR}($ ApIn $=$ number of apoptotic cells in the microscopic section / total cells in the microscopic section).

The statistical analysis was performed with the Mann-Whitney test due to the paired structure of the data and in order to compare variations, such as diabetic rats versus non-diabetic rats, one ESW versus two ESW and no ESW versus one or two ESW. A $p$ value $<0.05$ was considered to be statistically significant.

\section{RESULTS}

There was a significant increase in albumin urinary excretion $(\mathrm{p}<0.05)$ in all diabetic rats $(\mathrm{A} 1$, $\mathrm{A} 2, \mathrm{~A} 3)$ in comparison with the non diabetic ones (B1, B2, B3) as shown in Table-1.
The data in Table-2 demonstrated a significant difference with the diabetic animals $(\mathrm{p}=3.38 *)$ in the ApIn between groups A1 (no ESW) and A3 (2 ESW). On the other hand, ESW sub-group A2 (one ESW) showed a significantly less ApIn $(\mathrm{p}=2.84 *)$ than A3 (2 ESW). There were no significant differences between all sub-groups in the non-diabetic rats.

\section{COMMENTS}

In the last few years, the predominant management of urolithiasis was extracorporeal shock wave lithotripsy (ESWL). The changes in technology have led to a four-fold increase in procedural re-admissions within 30 days of primary separation (14). Renal parenchyma damage and the exact time between the first and any repeated ESW treatments is controversial, especially when associated with another prevalent disease such as diabetes (3-6). In this study, repeated ESW at 14-day intervals did not increase the apoptosis index in the renal parenchyma of normal rats. On the other hand, the second ESW in the diabetic rats was associated with a statistically significant increase in the apoptotic index (ApIn).

Research in lithotripsy reports the effort to characterize acute shock wave damage to the kidney. Different conditions in humans $(1-3,9,13,14)$ and experimental animals like rabbits (15), dogs (4, 6, $16)$, pigs $(2,17)$ and rats $(5,11)$ confirm the ESW injury on the renal parenchyma. Some factors can

Table 1 - Distribution of albumin value $(\mu \mathrm{g} / L)$ dosages in 24-hour urine taken just before the first ESW. Values are given as mean $\pm S E M$. All animals from group A were diabetics induced by alloxan and subjected to zero (A1), one (A2) or two (A3) ESW. All animals from group B were non-diabetics rats also subjected to zero (B1), one (B2) or two (B3) ESW. Different superscript letters indicate statistically significant differences in the corresponding (AI or B1) value in each group (Mann-Whitney, Z crit $=2.12$ ).

\begin{tabular}{lccccccc}
\hline & \multicolumn{3}{c}{ Group A (Diabetes) } & \multicolumn{3}{c}{ Group B (No Diabetes) } \\
& $\mathbf{A 1}$ & $\mathbf{A 2}$ & $\mathbf{A 3}$ & $\mathbf{B 1}$ & $\mathbf{B 2}$ & $\mathbf{B 3}$ \\
\hline Mean & $30.3^{\mathrm{a}}$ & $31.7^{\mathrm{b}}$ & $30.3^{\mathrm{c}}$ & $6.3^{\mathrm{a}}$ & $6.2^{\mathrm{b}}$ & $7.2^{\mathrm{c}}$ \\
SEM & 00.4 & 00.3 & 00.4 & 0.3 & 0.4 & 0.3 \\
Median & 25.0 & 25.0 & 25.0 & 5.7 & 5.5 & 6.7 \\
\hline
\end{tabular}

${ }^{a}=A 1>B 1(Z c a l=3.74 *),{ }^{b}=A 2>B 2(Z c a l=3.66 *),{ }^{c}=A 3>B 3(Z c a l=3.84 *)$ 
Table 2 - The apoptotic index calculated using the Image Pro-Plus V computer program shows the imunohistochemical expression of caspase-3. All animals from group A were diabetics induced by alloxan and subjected to zero (A1), one (A2) or two (A3) ESW. All animals from group B were non-diabetics rats who were also subjected to zero (B1), one (B2) or two (B3) ESW. Different superscript letters indicate statistically significant differences in to the corresponding (AI or B1) value in each group. Mann-Whitney test $(Z$ crit $=2.12)$.

\begin{tabular}{|c|c|c|c|c|c|c|}
\hline & \multicolumn{3}{|c|}{ Group A (Diabetes) } & \multicolumn{3}{|c|}{ Group B (No Diabetes) } \\
\hline & A1 & A2 & $\mathbf{A 3}$ & B1 & B2 & B3 \\
\hline Mean & $7.6^{\mathrm{a}}$ & $7.4^{\mathrm{b}, \mathrm{d}}$ & $11.9^{\mathrm{a}, \mathrm{b}, \mathrm{c}, \mathrm{d}}$ & 7.9 & 8.4 & $9.1^{\mathrm{c}}$ \\
\hline SEM & 0.2 & 0.3 & 0.4 & 0.2 & 0.2 & 0.3 \\
\hline Median & 6.8 & 7.0 & 12.5 & 8.0 & 7.0 & 9.0 \\
\hline
\end{tabular}

No ESW x two ESW: $A 1=B 1(\mathrm{Zcal}=0.68), A 1=A 2(\mathrm{Zcal}=0.78),{ }^{a}=A 1<A 3\left(\mathrm{Zcal}=3.38^{*}\right), B 1=B 2=B 3(\mathrm{Zcal}=0.72)$. Non diabetes $x$ diabetes: $A 1=B 1(\mathrm{Zcal}=1.48) ;{ }^{b}=A 2<A 3\left(\mathrm{Zcal}=3.83^{*}\right), A 2=B 2(\mathrm{Zcal}=1.75) ;{ }^{c} A 3>B 3\left(\mathrm{Zcal}=3.86^{*}\right)$. One ESWx Two ESW: ${ }^{d}=A 3>A 2\left(Z \mathrm{cal}=2.84^{*}\right), B 2=B 3(\mathrm{Zcal}=1.14)$.

produce contradictory results, including the sample size, different equipment with different energy principles, the number of impulses and the focus size (6). The present experimental model with a small animal such as a rat would show a proportionally larger area of the renal parenchyma affected by the shock wave in comparison to humans; however, a proportional dose of energy for lithotripsy (2,000 ESW) as used in humans was used. The area affected by the shock wave in the kidney, referred to as the focal point, was $11 \times 9 \mathrm{~mm}$ with the lithotriptor employed, corresponding to approximately $10 \%$ of the renal tissue exposed in human kidneys, and almost the entire parenchyma in rats (6).

Diabetes is a disease that promotes morphological and biochemical changes $(7,8)$ that worsen renal parenchyma conditions and induce etiologic impairment in the follow-up of urolithiasis treatment (18). The present experimental model of diabetes induced by alloxan within 30 days (12) promoted diabetic nephropathy, which were confirmed by the 24 hour microalbuminuria values (19) (Table 1) and the mesangial impairment on the histological sections described elsewhere (5).

The severity of the renal injury caused by the ESW is related to the number of shock waves administered and demonstrates the connection between renal structure and function damage (2). Beginning after four hours of supraclinical doses of $\operatorname{ESW}(8,000)$ in porcine, the glomerular filtration and renal plasma flow remained impaired over the following 24-hour period (2). In a prospective longitudinal study carried out in 14 patients with normal renal function subjected to extracorporeal shock waves lithotripsy (ESWL), renal function recovered on the $4^{\text {th }}$ day after ESWL, and there were no significant differences in the localization of the stones (19). Considering those points, we chose for our study the $3^{\text {rd }}$ day after ESW for the impact on the ApIn of renal parenchyma. Our choice of conducting the second ESW on the $14^{\text {th }}$ day following the first ESW was based on a sufficient time interval for recovery of damage from the first treatment.

The formation of free radicals plays a major role in shock wave lithotripsy induced renal damage. Moreover, previous studies suggest that free radicals may also promote de novo calcium oxalate crystallization of previously damaged urothelium (20). The shock wave trauma is primarily a vascular lesion and the injury is dose dependent. Vasoconstriction and parenchyma hemorrhage can be lead to a permanent loss in functional renal mass $(9,10,19)$. Secondary to vascular events, the hypoxia promotes a biochemical cellular impairment that, beyond necrosis, initiated the activation of caspase cascade of apoptosis $(10,18$, 19). Oxidative stress and hypoxia-induced changes are one of several key problems of endothelial cell pathobiology relevant to chronic kidney disease (10, $18,19)$. 
Caspase-3 plays a key role in apoptosis, and it has been reported that kidneys with ischemia showed increased apoptosis in tubular and interstitial areas compared with control kidneys (10). The caspases participate in important pathogenic mechanisms of apoptosis due to vasoconstriction and hypoxia. The early inhibition of caspases attenuates these mechanisms and reduces renal function impairment (11). Apoptosis and caspase- 3 are not the only event involved in ischemia injury, but as a biological marker the latter can act in monitoring and predicting the risk of functional renal impairment $(10,11)$. Apoptosis and caspase- 3 are also involved in long-term renal I/ $\mathrm{R}$ injury $(10,11)$. We suggest that the eventual association of ESW and apoptosis can be useful in monitoring the employment of procedures and drugs to protect the harmful effects of ESW (20), especially in pathological situations with previous vascular damage, like diabetes.

The results of the present work showed that the ApIn in normal rats with one ESW $(8.4 \pm 0.2)$ or two ESW $(9.1 \pm 0.3)$ was quite similar to the ApIn of animals with no ESW $(7.9 \pm 0.2)$. The shock waves did not increase the caspase- 3 expression in non-diabetic rats and this suggests that the oxidative stress does not play an essential role in the well-known transient structural and functional damage of the renal parenchyma in normal rats.

On the other hand, in a previous study of compromised renal tissue in diabetic rats, the ApIn with one ESW $(7.4 \pm 0.3)$ was similar to that with diabetic rats with no ESW and similar to normal rats with one or two ESW. One simple shock wave to the renal parenchyma of diabetic rats had the same impact as a single or double ESW in normal rats. However, with two ESW (11.9 \pm 0.4$)$, the ApIn was higher statistically significant in comparison with one ESW (7.4 \pm 0.3$)$.

Our experimental results suggested that diabetes was a condition that could increase the risk of renal injury in treatment with repeated ESW. Within the limitations of the model, a repeated ESW should be considered a procedure of additional risk in a diabetic as opposed to a healthy person. Considering that the damage is dose-dependent, divided ESW treatment at a larger frequency rate is advisable in a clinical setting with diabetic patients.

\section{CONCLUSIONS}

The apoptosis index on renal parenchyma in normal rats with a single $(2,000 \mathrm{SW})$ or repeated ESW $(4,000 \mathrm{SW})$ was similar to the control animals without any ESW. In the diabetic rat, the two ESW sessions $(4,000 \mathrm{SW})$ may result in an additional risk of tissue damage, which is evidenced in the higher apoptosis index.

\section{CONFLICT OF INTEREST}

None declared.

\section{REFERENCES}

1. Moe OW: Kidney stones: pathophysiology and medical management. Lancet. 2006; 367: 333-44.

2. Willis LR, Evan AP, Connors BA, Shao Y, Blomgren PM, Pratt JH, et al.: Shockwave lithotripsy: dose-related effects on renal structure, hemodynamics, and tubular function. J Endourol. 2005; 19: 90-101.

3. Pienkny AJ, Streem SB: Simultaneous versus staged bilateral extracorporeal shock wave lithotripsy: longterm effect on renal function. J Urol. 1999; 162: 1591-3.

4. Koga H, Matsuoka K, Noda S, Yamashita T: Cumulative renal damage in dogs by repeated treatment with extracorporeal shock waves. Int J Urol. 1996; 3: 134-40.

5. Kira VM, Kaufmann OG, Ortiz V, Srougi M: Morphological alterationsfollowing of retreatment eletrohidraulic shock waves in rat kidney. J Endourol. 2000; 14 (Suppl 1): A6 (BS2-2).

6. Fortes MA, Andriolo A, Ortiz V, Srougi M: Effect of shock wave reapplication on urinary $\mathrm{N}$-acetyl-betaglucosaminidase in canine kidney. Int Braz J Urol. 2004; 30: $148-54$.

7. Mbarki M, Jabrane J, Oussama A, Daudon M: Study of crystalluria in diabetic patients. Prog Urol. 2005; 15: 420-5; discussion 425-6.

8. Krambeck AE, Gettman MT, Rohlinger AL, Lohse CM, Patterson DE, Segura JW: Diabetes mellitus and hypertension associated with shock wave lithotripsy of renal and proximal ureteral stones at 19 years of followup. J Urol. 2006; 175: 1742-7.

9. McAteer JA, Bailey MR, Williams Jr JC, Cleveland RO, Evan AP: Strategies for improved shock wave lithotripsy. Minerva Urol Nefrol. 2005; 57: 271-87. 
10. Haase VH: Hypoxia-inducible factors in the kidney. Am J Physiol Renal Physiol. 2006; 291: F271-81.

11. Homsi E, Janino P, de Faria JB: Role of caspases on cell death, inflammation, and cell cycle in glycerol-induced acute renal failure. Kidney Int. 2006; 69: 1385-92. Erratum in: Kidney Int. 2006; 70: 1666.

12. Seidel AC, Fagundes DJ, Bazotte RB, Novo NF, Juliano Y, Meister H: Effect of lung resection and sham surgery on the frequency of infection in alloxan-diabetic rats. Braz J Med Biol Res. 2003; 36: 287-90.

13. Frang D, Hamvas A, Kalman J, Panovics J, Hegedus M, Szucs M, et al.: Experience with the Direx Tripter X1 shock-wave lithotripter. Int Urol Nephrol. 1992; 24: 481-90.

14. Holman CD, Wisniewski ZS, Semmens JB, Bass AJ: Changing treatments for primary urolithiasis: impact on services and renal preservation in 16,679 patients in Western Australia. BJU Int. 2002; 90: 7-15.

15. Li X, He D, Zhang L, Cheng X, Sheng B, Luo Y: A novel antioxidant agent, astragalosides, prevents shock wave-induced renal oxidative injury in rabbits. Urol Res. 2006; 34: 277-82.
16. Block G, Adams LG, Widmer WR, Lingeman JE: Use of extracorporeal shock wave lithotripsy for treatment of nephrolithiasis and ureterolithiasis in five dogs. J Am Vet Med Assoc. 1996; 208: 531-6.

17. Sheir KZ, Lee D, Humphrey PA, Morrissey K, Sundaram CP, Clayman RV: Evaluation of synchronous twin pulse technique for shock wave lithotripsy: in vivo tissue effects. Urology. 2003; 62: 964-7.

18. Pak CY, Sakhaee K, Moe O, Preminger GM, Poindexter JR, Peterson RD, et al.: Biochemical profile of stoneforming patients with diabetes mellitus. Urology. 2003; 61:523-7.

19. Cancho Gil MJ, Diz Rodriguez R, Virseda Chamorro M, Alpuente Roman C, Cabrera Cabrera JA, Panos Lozano P: A comparative study of the renal damage produced after the extracorporeal shock wave lithotripsy according to the lithiasis location. Actas Urol Esp. 2005; 29: 373-7.

20. Delvecchio FC, Brizuela RM, Khan SR, Byer K, Li Z, Zhong P, et al.: Citrate and vitamin E blunt the shock wave-induced free radical surge in an in vitro cell culture model. Urol Res. 2005; 33: 448-52.

\author{
Correspondence address: \\ Dr. Djalma José Fagundes \\ Rua Camé, 242, conjunto 33, $3^{\circ}$. andar \\ São Paulo, SP, 03121-020, Brazil \\ E-mail: djfagundes.dcir@epm.br
}

